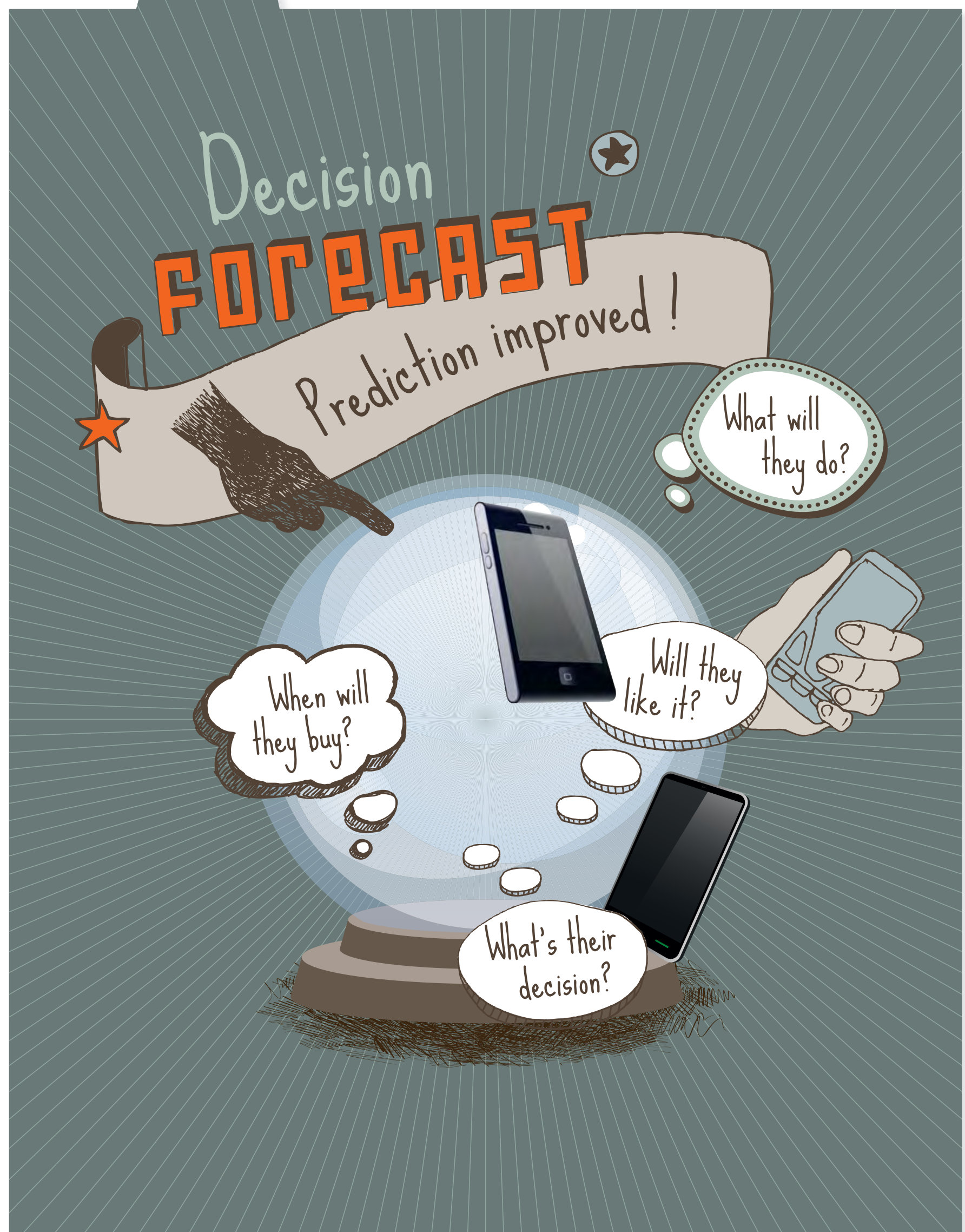




\title{
I Will ... Sooner or Later PREDICTING WHETHER AND WHEN CONSUMERS INTEND TO ADOPT NEW TECHNOLOGIES
}

\author{
Koert van Ittersum and Fred M. Feinberg
}

Two of the most critical uncertainties associated with new technology introductions are whether and when the target market will adopt them. A new scale integrates cumulative time intervals and predicts adoption more accurately. Behavioral data collected during a two-year longitudinal study provides empirical evidence for its accuracy. The new measure outperformed two single-intent measures and achieved a hit rate of more than $80 \%$ in predicting whether and when a cell-phone technology was adopted. Adoption likelihood can be estimated without actual sales data and thus be determined prior to the launch of a new product.

\section{The Challenge of Predicting Adoption Intentions Accurately}

Two of the most critical uncertainties associated with new-technology introductions are whether and when the target market will adopt them. Both uncertainties pose serious challenges for marketing managers planning a technology's production, pricing, distribution, and promotion. One way to reduce these uncertainties is to survey the target market about their intentions to adopt the technology. Because self-reported adoption intentions can be collected prior to launch at relatively low cost, they are among the most widely applied proxy measures for actual adoption.

Intention measures do correlate with actual behavior, but their predictive accuracy is rather limited. Indeed, the probability that people follow through on their intentions is quite modest: their intentions might change over time, intentions are not tightly interrelated with actual behavior, or the intention measure itself might be biased.
Two general approaches address biases that limit the predictive accuracy of intention measures. The biases are either accounted for in a formal statistical model or the measurement itself aims to reduce biases when intentions are first collected. While correcting for biases via dedicated statistical models can improve predictive accuracy of self-reported intentions, reducing them in the first place decreases the need for such complex approaches. Furthermore, actual sales data is often required for calibration purposes, reducing the practical value of many of these models.

\section{Measuring Timed Purchase Likelihood}

Including time in the measurement of adoption intentions can help better predict important managerial metrics. A new response scale collects self-reported adoption intentions prior to the launch of a new product and provides more accurate predictions of whether

\section{THE AUTHORS}

Koert van Ittersum, Associate Professor of Marketing, Scheller College of Business, Georgia Institute of Technology, Atlanta, USA, koert.vanittersum@ scheller.gatech.edu

Fred M. Feinberg, Phelps Collegiate Professor of Management, Stephen M. Ross School of Business, University of Michigan, Ann Arbor, USA, feinf@umich.edu

The article is an abridged version of Van Ittersum, Koert; Feinberg, Fred M. (2010): "Cumulative Timed Intent: A New Predictive Tool for Technology Adoption", Journal of Marketing Research, Vol. 47 (Oct.), pp. 808 - 822, and is published with the permission of the American Marketing Association. 
$\{$ Box 1 $\}$

\section{THE STUDY}

354 students of a large U.S. university participated in a study on their intentions to adopt cell phones with GPS technology. After the participants entered the lab, they were informed that a growing number of companies were introducing cell phones with GPS technology and that the researchers were interested in their intent to buy one. Half the participants were asked to express their intent to adopt using two traditional single-intent measures (scaled intention measure and open-ended information of when they expect they will have purchased the GPS cell phone), while the other half was asked to express their intent to adopt using cumulative timed intent measures.

A 36-month time horizon was selected, based on an average cell phone replacement rate of 24 months. Cumulative timed intent was measured among half the sample using 3-month increments. The response scale ranged from $0 \%=$ "I will not have bought one" to $100 \%=$ "I will have bought one", with $10 \%$ intervals. The $10 \%$ interval width was selected as a trade-off between scale granularity and the potential for being taxing to respondents. Of the participants, $40.4 \%$ (about half for each type of measurement) participated in four follow-up surveys (every six months over the following two years).

The estimation was carried out according to the general linear model and hierarchical Bayes methods. The main idea was to have a standard heterogeneous linear regression formulation underlie the response scale. This stage maps covariates adoption propensities that account for each subject's time trend. In a second stage, these propensities are mapped onto a probability scale using a probit transform. The third stage maps these probabilities to stated intentions, using a "multiplicative" or "rank order" binomial specification. As such, it is the first of these stages that completely accounts for individualspecific covariates' effects on adoption propensity; the two later stages simply transform these propensities to probabilities and then to intentions scale points. a future behavior will occur plus when this behavior is most likely (see Table 1). The scale specifies a clear and technology-specific time horizon (e.g., 36 months). This time horizon is divided into shorter, cumulative time intervals (e.g., $0-3$ months, $0-6$ months, ..., $0-36$ months). Then, respondents provide adoption probabilities for each cumulative time interval (e.g., 0-100\%). Asking for subjective probabilities in specified time frames encourages and allows respondents to better account for future uncertainty than asking them for their intentions alone does. As a consequence, subjective probabilities should more accurately predict future behavior than intentions when the behavior becomes distal in time.

In an experiment (Box 1 ) the ability of this scale to predict whether and when the technology would be adopted was compared to single intent measures.

\section{Predicting Adoption Accurately}

Figure 1 compares how well the different approaches predicted whether decision-makers would adopt a cell phone with GPS technology during the first two years of technology availability.

The self-reported probability curve of the cumulative timed intent measures proved to be highly accurate: it correctly classified $87.2 \%$ of the consumer decisionmakers as non-adopters and $80.6 \%$ as adopters, with an overall "hit rate" of $84.3 \%$, and it substantially outperformed the single intent measures. Particularly for

\section{») Asking for subjective probabilities in} specified time frames encourages and allows respondents to better account for future uncertainty than asking them for their intentions alone does. « 


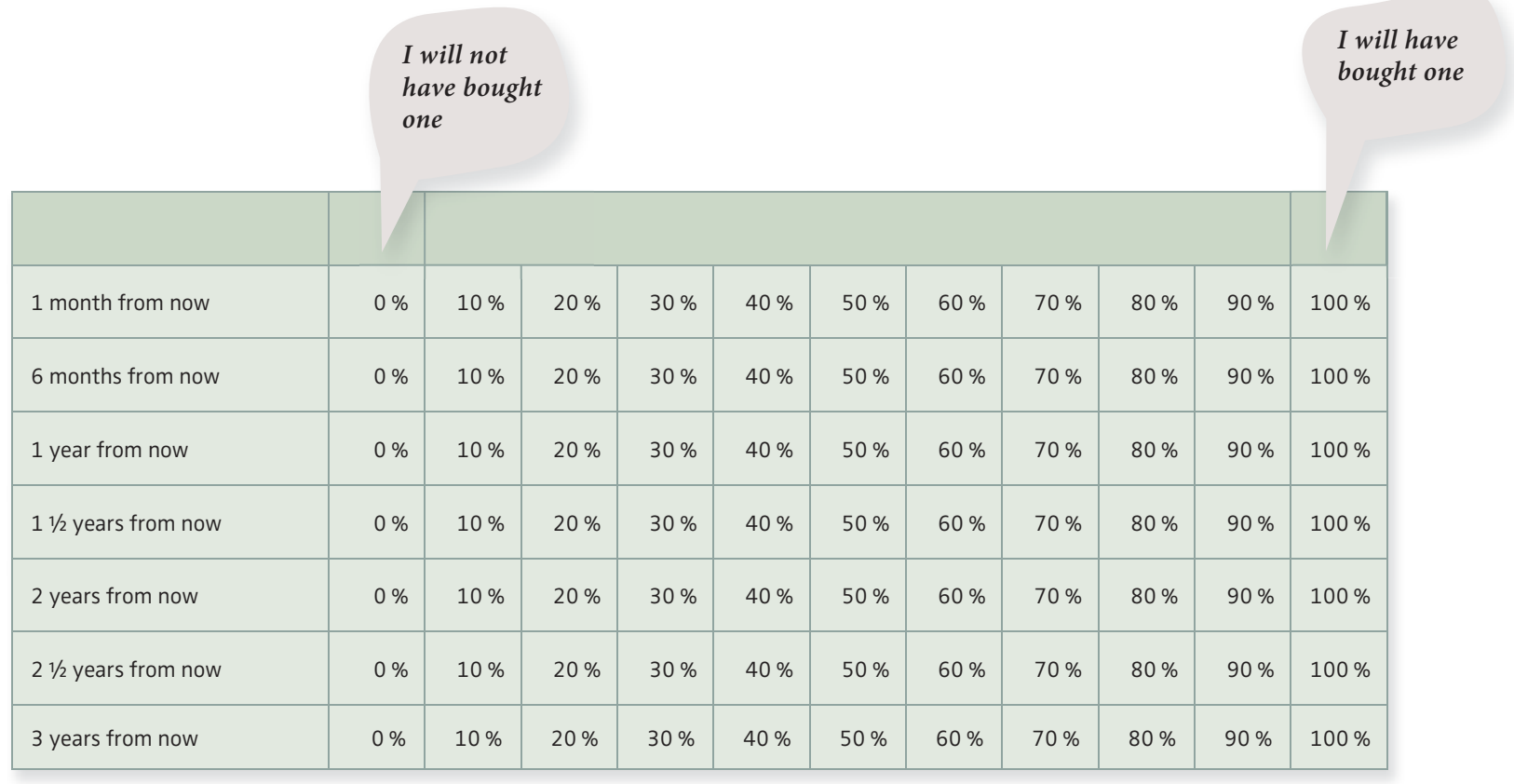

adopters, the hit rates of a traditional single intent measure and the open-ended responses of whether and when respondents intend to adopt the new technology were significantly lower. Therefore, the particular strength of the cumulative timed intent measures lies in correctly classifying adopters, which, generally speaking, is more elusive than predicting the status quo of non-adoption.

A comparison of the absolute differences in the predicted and actual timing of adoption confirms the superior performance of cumulative timed intent measures. It most accurately predicted the timing of adoption: for a 24-month time period, the mean deviation of the predicted time of adoption is a relatively small 3.6 months, compared to 5.5 months for the single intent and 8.8 months for the self-report measure (Figure 2).

\section{Application in Practice}

The big advantage of this approach is that individuallevel cumulative adoption likelihood curves can be estimated without actual sales data, and thus can be determined prior to launch. Accurately predicting whether and when people intend to adopt a relevant technology reduces the uncertainty of whether and when the target market will adopt a new technology, allowing marketing managers to better plan a technology's production, pricing, distribution, and promotion.

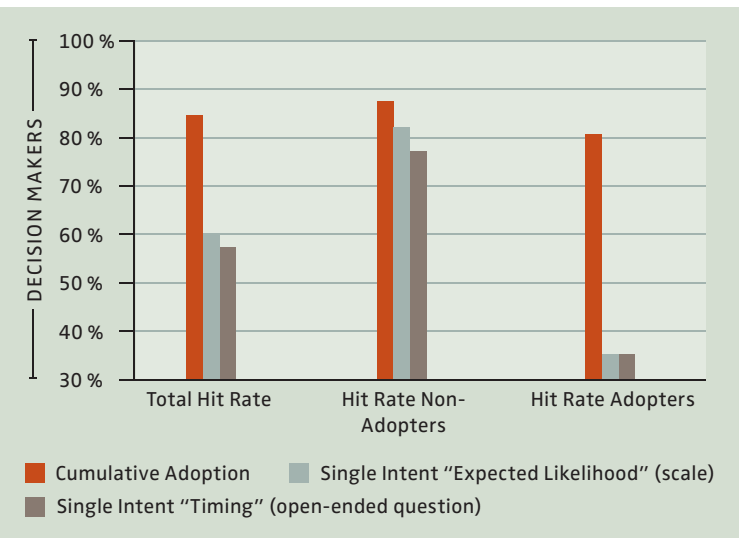

FIGURE 1:

Individual Level Adoption Hit Rate of the Adoption of a Cell Phone with GPS Technology

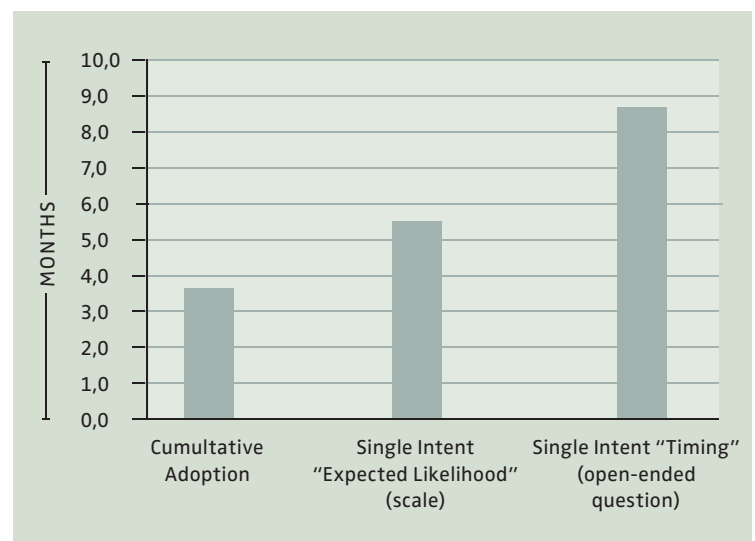

\section{FIGURE 2:}

Timing of Adoption Mean Absolute Deviation of Cumulative Time Intent Measure (Months) 
FURTHER READING

\section{») The particular strength of the}

\section{cumulative timed intent measures}

lies in correctly classifying adopters,

which, generally speaking, is more

elusive than predicting the status

quo of non-adoption. «
Besides collecting intentions to predict first-time purchase behavior, intentions can also be collected to predict repeat purchases. For instance, using satisfaction ratings, companies often try to predict customer repurchase intentions. The proposed cumulative timed intent measure may mitigate some of the challenges faced when collecting satisfaction and intent data in the same survey. It allows a closer and more accurate examination of the relationship between satisfaction and future behavior. •
Castaño, Raquel, Mita Sujan, Manish Kacker, and Harish Sujan (2009): "Preparing for the Adoption of the New Arrival", GfK Marketing Intelligence Review, Vol. 1, No. 2 (November), $16-23$.

Hsiao, Cheng, Baohong Sun, and Vicki G. Morwitz (2002), "The Role of Stated Intentions in New Product Purchase Forecasting", Econometric Models in Marketing, 16, $11-28$.

Morwitz, Vicki G., Joel H. Steckel, and Alok Gupta (2007) "When Do Purchase Intentions Predict Sales?" International Journal of Forecasting, 23 (3), $347-64$.

\section{KEYWORDS:}

Forecasting Methods, Technology Adoption, (Re-)Purchase Intentions, Innovation Management 